\title{
Propofol and Remifentanil Sedation for Bronchial Thermoplasty: A Prospective Cohort Trial
}

\author{
Julia N.S. d'Hooghe ${ }^{a}$ Susanne Eberl ${ }^{b}$ Jouke T. Annema ${ }^{a}$ Peter I. Bonta ${ }^{a}$ \\ Departments of a Pulmonology and ${ }^{b}$ Anesthesiology, Academic Medical Center, University of Amsterdam, \\ Amsterdam, The Netherlands
}

\section{Keywords}

Bronchial thermoplasty $\cdot$ Sedation $\cdot$ Propofol $\cdot$ Asthma .

Bronchoscopy

\begin{abstract}
Background: Bronchial thermoplasty (BT) is a rapidly emerging bronchoscopic treatment for patients with moderate-tosevere asthma. Different sedation strategies are currently used, ranging from mild midazolam sedation to general anesthesia requiring tracheal intubation. Objectives: The aim of this study was to assess the feasibility, safety, and both patients' and bronchoscopists' satisfaction with propofol and remifentanil sedation administered by specialized sedation anesthesiology nurses during BT in severe asthma patients. Methods: A prospective observational cohort study in BT-treated severe asthma patients of the TASMA trial was designed. Patients were asked to rate their overall BT procedure satisfaction and tolerance with propofol/remifentanil sedation using a visual analogue scale (VAS) ranging from 0 to 10 . Similarly, bronchoscopists were asked to rate patient cooperation and tolerance. Sedation-associated adverse events and the number of BT activations were recorded. $\boldsymbol{R e}$ sults: Thirty-two BT procedures in 13 severe asthma patients were performed under moderate target-controlled infusion (TCl) propofol/remifentanil sedation. Patients' median VAS scores were as follows: overall satisfaction 9.6 (interquartile
\end{abstract}

\section{KARGER}

(c) 2016 S. Karger AG, Basel

E-Mail karger@karger.com

www.karger.com/res range [IQR] 8.5-10.0), dyspnea 0.0 (IQR 0.0-0.6), pain 0.1 (IQR $0.0-1.0$ ), cough 0.5 (IQR 0.0-2.1), and anxiety 0.1 (IQR $0.0-$ 0.7). Bronchoscopists' median VAS scores were as follows: overall patient cooperation 9.1 (IQR 8.5-9.6), dyspnea 0.3 (IQR 0.0-0.9), pain 0.2 (IQR 0.0-1.3), cough 1.2 (IQR 0.7-2.0), and discomfort 0.6 (IQR 0.3-1.5). All patients were willing to undergo the procedure again and would recommend this form of sedation to their best friend. One case of conversion to general anesthesia occurred and no serious adverse events were reported. Conclusions: Moderate sedation with propofol and remifentanil TCl provided by specialized sedation anesthesiology nurses is feasible and safe and results in high satisfaction rates of both patients and bronchoscopists.

(c) 2016 S. Karger AG, Basel

\section{Introduction}

Bronchial thermoplasty (BT) is a novel bronchoscopic treatment for moderate-to-severe asthma patients that has been shown to improve quality of life and to reduce asthma symptoms and exacerbation rates [1-3]. Although the mechanism of action of BT is largely unknown, there is evidence that $\mathrm{BT}$ reduces airway smooth muscle mass

J.N.S. d'Hooghe and S. Eberl contributed equally to this work.
Peter I. Bonta, MD, PhD

Department of Pulmonology, Academic Medical Center University of Amsterdam, F5-144, Meibergdreef 9 NL-1105 AZ Amsterdam (The Netherlands)

E-Mail p.i.bonta@amc.nl 
$[4,5]$. A complete BT treatment consists of 3 consecutive bronchoscopy procedures during which airways of the left lower lobe, right lower lobe, and finally both upper lobes are treated. During BT treatment, radio-frequent energy is delivered to the larger airways by a 4-strut expandable basket catheter that heats the surrounding tissue during $10 \mathrm{~s}$ to $65^{\circ} \mathrm{C}$, a so-called activation [6]. On average, between $\sim 50$ and $\sim 75$ activations are delivered to treat all reachable airways with a diameter of 3-10 $\mathrm{mm}$.

Since BT treatment is performed in severe asthma patients with highly reactive airways and since BT procedures are time-consuming and require high precision, these treatments were initially performed under general anesthesia [3]. To date, there is a large variety of sedation forms used during BT procedures. Martin et al. [7] reported mild sedation with midazolam and fentanyl. Lee et al. [8] described monitored anesthesia care with dexmedetomidine. Other strategies include deep propofol sedation or general anesthesia requiring tracheal intubation or laryngeal mask insertion [9]. The drawbacks of mild midazolam sedation are cough and patient discomfort [10] for an interventional procedure which needs to be performed 3 times. On the other end of the spectrum, general anesthesia with intubation could be seen as overtreatment, since this is more invasive, is associated with higher complication risks, including hemodynamic instability, and requires a more complex infrastructure with higher costs. Propofol and remifentanil sedation have been known to be safe and feasible for standard flexible bronchoscopies, thoracoscopy, and other bronchoscopic procedures, including endobronchial ultrasound bronchoscopy [11-14]. Considering the above, with the current implementation of $\mathrm{BT}$, there is an unmet need for a feasible, safe, and cost-effective sedation method for BT with high patient and bronchoscopist satisfaction. In this study, we evaluated whether for BT moderate sedation with target-controlled infusion (TCI) of propofol combined with remifentanil provided by specialized sedation anesthesiology nurses is feasible, safe, and satisfying - for both patients and bronchoscopists.

\section{Methods}

\section{Study Design}

The current sedation study is an observational cohort study within the Unravelling Targets of Therapy in Bronchial Thermoplasty in Severe Asthma (TASMA) trial (ClinicalTrials.gov, No. NCT02225392). BT procedures were performed by 2 experienced bronchoscopists (P.I.B. and J.T.A.) in the endoscopy intervention suite at the Department of Pulmonology, Academic Medical Center (AMC), Amsterdam, The Netherlands.

Sedation for Bronchial Thermoplasty

\section{Patients}

Patients with severe asthma aged between 22 and 61 years, participating in the TASMA trial, were included in the study. Severe asthma was defined as asthma that was symptomatic despite treatment with high-dose inhaled corticosteroids (dosage $\geq 500 \mu \mathrm{g}$ fluticasone or equivalent per day) and inhaled long-acting $\beta_{2}$ agonist (dosage of $\geq 100 \mu \mathrm{g}$ salmeterol or equivalent per day), with a bronchial hyperresponsiveness to methacholine (PC20<4 mg/ $\mathrm{mL}$ ), and that fulfilled the World Health Organization or modified Innovative Medicines Initiative criteria of severe refractory asthma $[15,16]$. Screening included a spiral computed tomography scan of the chest and a flexible bronchoscopy to exclude airway abnormalities including tracheomalacia. Exclusion criteria were a forced expiratory volume in $1 \mathrm{~s}$ percentage predicted $<50 \%$, a body mass index $>35$, and known allergy to bronchodilators, propofol, remifentanil, or other sedative medicine.

\section{Sedation Intervention}

In the AMC Amsterdam hospital (as well as in other hospitals in the Netherlands), endoscopy procedures are supported by specialized sedation anesthesiology nurses who perform self-reliant procedural sedations. They have followed a theoretical and practical sedation education program of 1 year that is completed by an examination that results in certification. These specialized sedation anesthesiology nurses provided the sedation with propofol and remifentanil and were responsible for continuous monitoring during the BT procedure in the endoscopy suite with an anesthesiologist on call in case of unexpected events.

Before the beginning of the BT procedure, a peripheral intravenous line was inserted, and glycopyrrolate $0.2 \mathrm{mg}$, lidocaine 20 $\mathrm{mg}$, and midazolam $1 \mathrm{mg}$ were administered. Sedation was started using a remifentanil TCI system (remifentanil [Ultiva] $2 \mathrm{mg}$ powder, GlaxoSmithKline BV, Philadelphia, PA, USA) starting with a targeted plasma level of $1.5 \mu \mathrm{g} / \mathrm{mL}$ combined with a propofol TCI system (propofol 1\%, MCT/LCT Fresenius, Zeist, The Netherlands) with a targeted plasma level of $1.2 \mu \mathrm{g} / \mathrm{mL}$. TCI means a weight- and age-adapted preprogramed system using a defined pharmacokinetic model to attain a specific blood target level. Enough time needs to be taken to achieve the adequate sedation level. Before introducing the bronchoscope, the level of sedation was assessed using the Observer's Assessment of Alertness/Sedation $(\mathrm{OAA} / \mathrm{S})$ score. When a score of 3 was reached, meaning that the patient responded only after his/her name was called loudly or repeatedly, the bronchoscope was introduced [17], and, additionally, lidocaine $1 \mathrm{mg} / \mathrm{kg}$ was administered on the vocal cords and the tracheal mucosa for local anesthesia. This local anesthesia administration was repeated every 10-15 min or when the patient started coughing with a maximum of $8.2 \mathrm{mg} / \mathrm{kg}$ [18]. During bronchoscopy, close communication between the bronchoscopist and the anesthesiology nurse was secured. In case of a desaturation, measures were taken, such as chin lift/jaw thrust, stimulating the patient to take a deep breath, temporary mask ventilation, or intubation. After the procedure, patients were transferred to the recovery room as soon as they reached a stable hemodynamic state with oxygen saturation $\left(\mathrm{SpO}_{2}\right)$ of $>92 \%$ without oxygen.

\section{BT Procedure and Monitoring}

Thirty minutes before the procedure, patients were nebulized with $0.5 \mathrm{mg} / 2.5 \mathrm{mg}$ ipratropium/salbutamol, respectively. BT procedures were performed with flexible bronchoscopes (Olympus, 
Table 1. Baseline characteristics

$\begin{array}{lc}\text { Subjects, } n & 13 \\ \text { Bronchial thermoplasty procedures, } n & 32 \\ \text { Gender, male/female } & 2 / 11 \\ \text { Age, years } & 42 \pm 14 \\ \text { Prebronchodilator } \mathrm{FEV}_{1} \text {, \% predicted } & 78 \pm 26 \\ \text { Inhaled corticosteroids of fluticasone or equivalent, } \mu \mathrm{g} / \text { day } & 1,269 \pm 525 \\ \text { Inhaled LABA of salmeterol or equivalent, } \mu \mathrm{g} / \text { day } & 154 \pm 97 \\ \text { Chronic oral corticosteroid use, } n & 6 \\ \text { Oral corticosteroids of prednisone or equivalent, } \mathrm{mg} / \text { day } & 13 \pm 7\end{array}$

Values are means \pm standard deviations unless otherwise indicated. $\mathrm{FEV}_{1}$, forced expiratory volume in $1 \mathrm{~s}$; LABA, long-acting $\beta_{2}$ agonist.
Tokyo, Japan) and with the ALAIR system (Boston Scientific, Natick, MA, USA) which automatically measures the number of successful activations per BT procedure. During the procedure, $2 \mathrm{~L} / \mathrm{min}$ of oxygen was administered by the Smart Capnoline (Medtronic Trading NL B.V., Eindhoven, The Netherlands) which enables exhaled carbon dioxide monitoring and nasal delivery of oxygen simultaneously. Patients were constantly monitored by specialized sedation anesthesiology nurses for heart rate, respiratory rate, $\mathrm{SpO}_{2}$, electrocardiogram, noninvasive blood pressure, and exhaled carbon dioxide measured continuously and recorded at 5-min intervals.

\section{Primary Endpoint}

The primary endpoint was satisfaction of patients and bronchoscopists. Patients were asked to rate their overall satisfaction by using a $10-\mathrm{cm}$ visual analogue scale (VAS; $0=$ "unbearable," $10=$ "excellent") as well as degree of dyspnea, pain, cough, and anxiety (VAS; 0 = "no complaints at all," 10 = "enormously") after they were fully awake. After the third BT procedure, patients were asked if they would be willing to undergo the entire procedure again under the same conditions and if they would recommend this form of sedation to their best friend. Similarly, directly after every BT procedure, the bronchoscopists were asked to score their satisfaction with sedation by rating the following aspects via a $10-\mathrm{cm}$ VAS: patient overall cooperation (VAS; $0=$ "unmanageable," $10=$ "excellent") and estimated degree of dyspnea, pain, cough, and discomfort for the patient (VAS; $0=$ "no complaints at all," $10=$ "enormously") [19].

\section{Secondary Endpoints}

As secondary endpoints the total procedure time (time between insertion and removal of the bronchoscope), sedation induction time (time between start of propofol/remifentanil infusion and insertion of the bronchoscope), time to sedation recovery (time between removal of the bronchoscope and arrival in the recovery room), time to discharge (time between removal of the bronchoscope and discharge from the recovery room), total amount of propofol and remifentanil dose, the number of successful BT activations per procedure, and the number of times backup by the anesthesiologist on call was needed were recorded. After finishing the procedure, the bronchoscopist scored the OAA/S scale to assess the overall level of sedation during the procedure.

\section{Sedation-Related Adverse Events}

Sedation-related adverse events were defined as a decline in $\mathrm{SpO}_{2}$ to $<90 \%$ during $>30$ s, hypotension or hypertension (blood pressure decreased/elevated $>20 \%$ of baseline blood pressure and requiring intervention), or respiratory arrest.

\section{Statistical Methods}

Descriptive statistics were performed, and results were expressed as means ( \pm standard deviations) in case of a normal distribution or variables, and medians (interquartile ranges [IQR]) were used for variables with a skewed distribution. Categorical data were expressed as numbers and percentages. Analysis was performed using SPSS software version 22 (IBM, Armonk, NY, USA).

\section{Statement of Ethics}

For the current prospective observational cohort sedation trial, ethical approval was obtained for the sedation questionnaires from the Medical Ethics Committee of the AMC, Amsterdam, The Netherlands (NL45394.018.13). All patients provided written informed consent.

\section{Results}

From August 2014 to April 2016, 13 patients underwent a total of $35 \mathrm{BT}$ procedures. Three out of the $35 \mathrm{BT}$ procedures were performed under general anesthesia. The other 32 procedures were performed under TCI propofol and remifentanil sedation and used for analysis. Baseline patient characteristics are summarized in Table 1 .

\section{Primary Endpoints}

The median VAS scores assessed by the patients were: overall satisfaction 9.5 (IQR 8.5-10.0) (Fig. 1), dyspnea 0.0 (IQR 0.0-0.6), pain 0.1 (IQR 0.0-1.0), cough 0.5 (IQR 0.0-2.1), and anxiety 0.1 (IQR 0.0-0.7) (Fig. 2a; Table 2). The mean VAS scores assessed by the bronchoscopists were: overall patient cooperation 9.1 (IQR 8.5-9.6)
60 
(Fig. 1), dyspnea 0.3 (IQR 0.0-0.9), pain 0.2 (IQR 0.01.3), cough 1.2 (IQR 0.7-2.0), and discomfort 0.6 (IQR 0.3-1.5) (Fig. 2b; Table 2). All patients were willing to undergo the $\mathrm{BT}$ procedures under the same conditions again and would recommend this form of sedation to their best friend (Table 2).

\section{Secondary Endpoints}

Secondary endpoints are summarized in Table 3 . The median total procedure time for BT procedures of the right, left, and both upper lobes was 43 (IQR 35-51) min, 46 (IQR 42-50) min, and 58 (IQR 51-65) min, respectively; time to sedation recovery was 6 (IQR 5-10) min; and time to discharge was 133 (IQR 103-169) min. A total of 433 (IQR 358-544) mg propofol and 517 (IQR 379850) $\mu$ g remifentanil were administered. During BT, a mean \pm standard deviation of $52 \pm 7,56 \pm 8$, and $75 \pm 18$ activations was given to the right, left, and both upper lobes, respectively. Backup by the anesthesiologist on call was needed twice. During the BT procedure, the majority of patients were able to open their eyes when called loudly and respond to assignments, such as "breathe deeply," and the most frequently rated OAA/S scores by the bronchoscopists were step 4 (48\%) and step 3 (42\%) (Table 4).

\section{Sedation-Related Adverse Events}

As shown in Table 5, no serious adverse events occurred. A total of 6 adverse events were reported. Respiratory adverse events, defined as desaturation $<90 \%$ during more than $30 \mathrm{~s}$, occurred 4 times. In 1 case of desaturation, it was decided to converse from moderate sedation with propofol and remifentanil to general anesthesia with tracheal intubation. The 3 other cases were restored with supportive care, such as chin lift and stimulating the patient to breathe deeply. The 2 nonrespiratory adverse events of hypotension were restored with administration of intravenous ephedrine. No other adverse events were encountered.

\section{Discussion}

In this study, we showed that moderate sedation with propofol and remifentanil TCI provided by specialized sedation anesthesiology nurses for BT in high-risk severe asthma patients is safe and feasible with high satisfaction rates for both patients and bronchoscopists. Therefore, this sedation strategy might qualify as an optimal strategy for BT since it has a fine balance between under- and oversedation that obviates the need for an anesthesiolo-

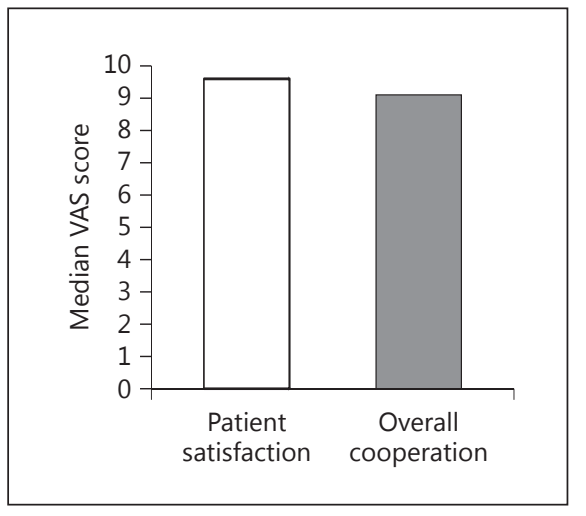

Fig. 1. Patients' and bronchoscopists' median VAS score for overall procedural satisfaction and overall patient cooperation. VAS, visual analogue scale.

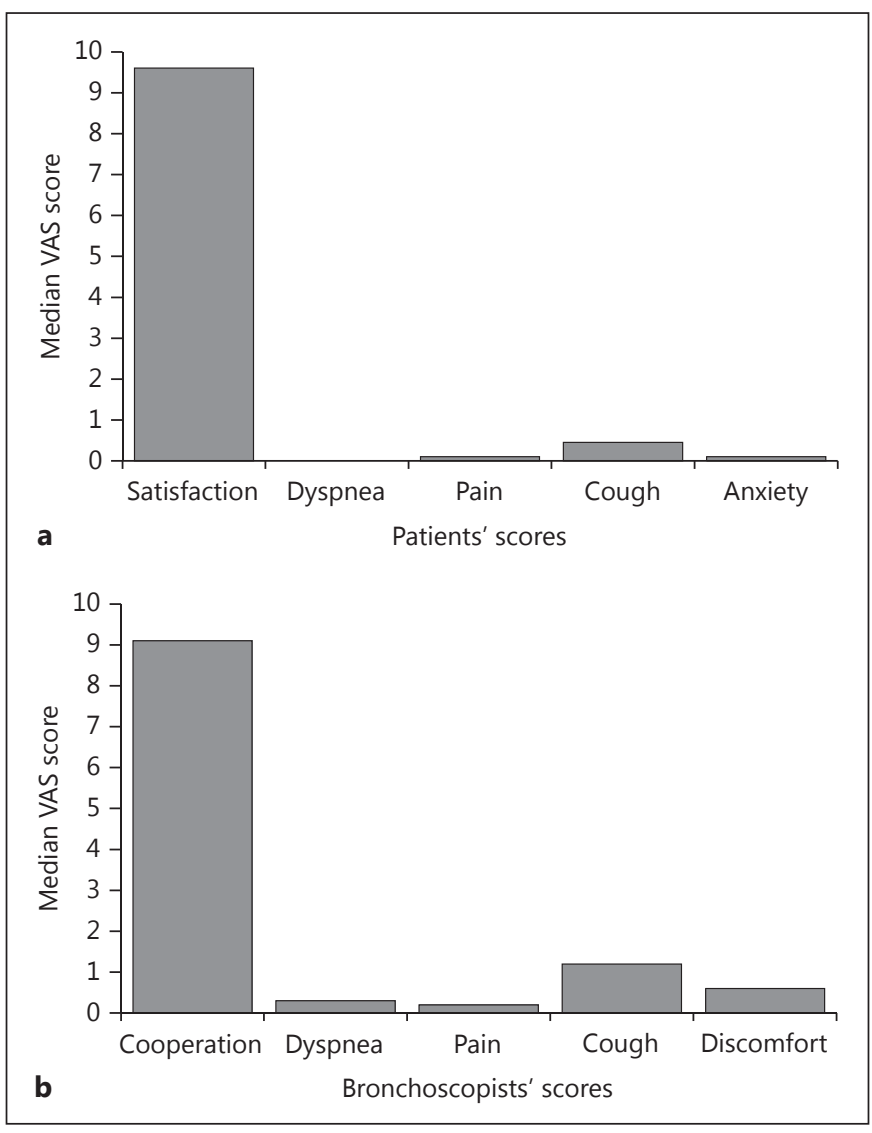

Fig. 2. Patients' and bronchoscopists' median VAS scores. a Patients' VAS scores for overall procedural satisfaction, dyspnea, pain, cough, and anxiety during BT procedures with propofol and remifentanil TCI sedation. b Bronchoscopists' median VAS scores for overall patient cooperation, dyspnea, pain, cough, and discomfort during BT procedures with propofol and remifentanil TCI sedation. VAS, visual analogue scale; BT, bronchial thermoplasty; TCI, target-controlled infusion. 
Table 2. Primary outcome parameters: patients' and endoscopists' assessment

\begin{tabular}{|c|c|}
\hline \multicolumn{2}{|l|}{ The median VAS scores as assessed by patients $(n=32)$} \\
\hline Overall satisfaction $^{\mathrm{a}}$ & $9.6(8.5-10)$ \\
\hline Dyspnea $^{\mathrm{b}}$ & $0.0(0.0-0.6)$ \\
\hline Pain ${ }^{b}$ & $0.1(0.0-1.0)$ \\
\hline Cough $^{\mathrm{b}}$ & $0.5(0.0-2.1)$ \\
\hline Anxiety ${ }^{\mathrm{b}}$ & $0.1(0.0-0.7)$ \\
\hline Willingness to repeat the BT procedures under the same conditions, $\%$ & 100 \\
\hline Recommendation of this form of sedation to their best friend, $\%$ & 100 \\
\hline \multicolumn{2}{|l|}{ The median VAS scores as assessed by the bronchoscopists $(n=32)$} \\
\hline Overall patient cooperation $^{c}$ & $9.1(8.5-9.6)$ \\
\hline Dyspnea & $0.3(0.0-0.9)$ \\
\hline Pain ${ }^{\mathrm{b}}$ & $0.2(0.0-1.3)$ \\
\hline Cough $^{\mathrm{b}}$ & $1.2(0.7-2.0)$ \\
\hline Discomfort ${ }^{\mathrm{b}}$ & $0.6(0.3-1.5)$ \\
\hline
\end{tabular}

Values are medians and interquartile ranges in parentheses. VAS, visual analogue scale; BT, bronchial thermoplasty. ${ }^{\text {a }}$ Defined as $0=$ "unbearable" and $10=$ "excellent." b Defined as $0=$ "no complaints at all" and $10=$ "enormously." c Defined as $0=$ "unmanageable" and $10=$ "excellent."

Table 3. Secondary outcome parameters $(n=32)$

\begin{tabular}{lc} 
Total procedure time, min & \\
BT 1 (right lobe) & $43(35-51)$ \\
BT 2 (left lobe) & $46(42-50)$ \\
BT 3 (upper lobes) & $58(51-65)$ \\
Sedation induction time, min & $18(13-20)$ \\
Time to sedation recovery, min & $6(5-10)$ \\
Time to discharge, min & $133(103-169)$ \\
Total propofol dose, mg & $433(358-544)$ \\
Total remifentanil dose, $\mu \mathrm{g}$ & $517(379-850)$ \\
\hline Number of activations & \\
BT 1 (right lobe) & $52 \pm 7$ \\
BT 2 (left lobe) & $56 \pm 8$ \\
BT 3 (upper lobes) & $75 \pm 18$ \\
Need for anesthesiologist backup, $n$ & 2
\end{tabular}

Values are medians and interquartile ranges in parentheses or means \pm standard deviations unless otherwise indicated. BT, bronchial thermoplasty.

gist to be present in the endoscopy suite. To our knowledge, we are the first to report on this specific sedation strategy successfully applied during BT procedures.

Propofol and remifentanil sedation has been known to be safe and feasible for standard flexible bronchoscopies, thoracoscopy, and other bronchoscopic procedures, including endobronchial ultrasound bronchoscopy [1114]. Several factors contribute to the importance of opti-
Table 4. Observer Assessment of Alertness/Sedation score

5 Responds readily to name spoken in normal tone $1(3 \%)$

4 Lethargic response to name spoken in normal tone $15(48 \%)$

3 Responds only after name is spoken loudly or rap- 13 (42\%) idly, or both

2 Responds only after mild prodding or shaking $1(3 \%)$

1 Does not respond to mild prodding or shaking $1(3 \%)$

0 Does not respond to noxious stimulus

$1(3 \%)$

Table 5. Adverse events $(n=32)$

Serious adverse events

0

Adverse events

Desaturation $\left(\mathrm{SpO}_{2}<90 \% \text { for at least } 30 \mathrm{~s}\right)^{\mathrm{a}}$

Hypotension (need for ephedrine)

Hypertension

Other

6
4
2
0
0

Values are numbers. $\mathrm{SpO}_{2}$, oxygen saturation. ${ }^{\text {a }}$ One case of desaturation required conversion to general anesthesia.

mal sedation for BT treatment in particular. First, BT procedures require high precision and last longer (43-58 min in total in this study) than diagnostic bronchoscopies. Second, they are performed in patients with highly reactive airways which involve an increased risk of developing laryngospasm and bronchospasm during bronchoscopy $[20,21]$. Every single activation during a BT represents a strong mechanical manipulation of the bronchial muco- 
sal wall that contributes to an increased risk of adverse events. Finally, the complete BT treatment consists of 3 separate BT procedures, and, thus, to fulfill the complete treatment, it is extremely important that the patient tolerates the procedure well.

We performed moderate sedation with propofol and remifentanil TCI combined with topical anesthesia facilitating a stable spontaneous breathing situation and, thus, obviating the need for intubation as well as muscle relaxant use and ventilator support. The feasibility of this sedation strategy was proven by high satisfaction levels combined with the fact that all BT procedures succeeded, with a total amount of activations that did not differ from the largest randomized controlled trial where a range of sedation types were used [22]. Previous studies have shown that VAS scores can be successfully applied to measure the level of satisfaction and its separate components $[19$, 23].

No serious adverse events occurred. The single patient in whom the investigated sedation strategy was converted to deep sedation was the first patient receiving this specific sedation protocol with propofol and remifentanil. In this first case, in retrospect, the initial dose of remifentanil chosen was too high, and insufficient time was taken for sedation induction, which resulted in apnea and rigidity of the thorax with desaturation. To avoid this problem in subsequent cases, sedation was started with a lower targeted plasma level of remifentanil $(1.5 \mu \mathrm{g} / \mathrm{mL})$, and sufficient time was taken to reach the optimal sedation level.

Propofol and remifentanil have the advantage of a rapid onset and termination of action which make this combination easily titratable. Remifentanil reduces cough and airway reactivity, resulting in a lower dose of propofol needed, and, therefore, reduces the risk of respiratory events $[24,25]$. Therefore, the sedation strategy used allows a rapid time to full recovery (in this study, a median time of $6 \mathrm{~min}$ ) allowing quick discharge from the hospital. Administration of propofol and remifentanil TCI requires skilled specialized sedation anesthesiology nurses or anesthesiologists experienced in the close monitoring of hemodynamic and respiratory parameters, such as $\mathrm{SpO}_{2}$, respiratory frequency, and end-expiratory $\mathrm{CO}_{2}$, since small dose modifications - especially of remifentanil - can highly impact the level of sedation, with a shift from moderate to deep sedation or even general anesthesia with the need to ventilate the patient. Currently, such sedation regimes are increasingly provided by specialized sedation anesthesiology nurses, since this is a recognized option according to the guidelines from the American Society of Anesthesiology [26-28]. However, propofol and remifentanil administration by specialized sedation anesthesiology nurses is still not common and/or available in many countries in Europe. Special educational programs combined with an optimal infrastructure, including an anesthesiologist on call, are required.

The strong point of the current study is the prospective design and the careful assessment of valid and important outcomes for both patients and bronchoscopists. Beside the moderate number of patients investigated, a limitation is the fact that 3 of the 35 procedures were performed under general anesthesia. We judge this potential selection bias to be small and regard it to be highly unlikely to affect the observed outcomes. Furthermore, no cost-effectiveness analysis was performed. A further limitation is the lack of a direct comparison to other sedation strategies (e.g., sedation with midazolam with or without [al]fentanyl or general anesthesia), since this study only investigates 1 specific sedation strategy, namely sedation with propofol and remifentanil TCI. Therefore, no conclusions can be drawn about the superiority of this sedation strategy over other sedation strategies, which have been successfully used in earlier clinical trials. Randomized controlled trials, preferably multicenter trials, that compare different sedation strategies are necessary to investigate the value of the different sedation strategies.

In conclusion, BT is a rapidly emerging bronchoscopic treatment for patients with severe asthma. Sedation with propofol and remifentanil TCI provided by specialized sedation anesthesiology nurses is feasible and safe and results in high satisfaction scores by both patients and bronchoscopists.

\section{Acknowledgements}

The authors would like to acknowledge and thank the specialized sedation anesthesiology nurses B. van Loo and A. van Lonkhuijzen and the endoscopy nurses of the AMC for their committed and professional work.

\section{Financial Disclosure and Conflicts of Interest}

Dr. P.I. Bonta and Dr. J.T. Annema received grants and material support from Boston Scientific to conduct the TASMA study (ClinicalTrials.gov; No. NCT02225392). Dr. P.I. Bonta has received honoraria from Boston Scientific for lectures about bronchial thermoplasty. The other authors declare no potential conflict of interest.

The TASMA study is funded by the Dutch Lung Foundation, The Netherlands Organization for Health Research and Development (ZonMw), and Boston Scientific. 


\section{References}

$>1$ Pavord ID, et al: Safety and efficacy of bronchial thermoplasty in symptomatic, severe asthma. Am J Respir Crit Care Med 2007;176: 1185-1191.

2 Castro M, et al: Effectiveness and safety of bronchial thermoplasty in the treatment of severe asthma: a multicenter, randomized, double-blind, sham-controlled clinical trial. Am J Respir Crit Care Med 2010;181:116-124.

$>3$ Cox G, et al: Asthma control during the year after bronchial thermoplasty. N Engl J Med 2007;356:1327-1337.

4 Pretolani M, et al: Reduction of airway smooth muscle mass by bronchial thermoplasty in patients with severe asthma. Am J Respir Crit Care Med 2014;190:1452-1454.

$\checkmark 5$ Miller JD, et al: A prospective feasibility study of bronchial thermoplasty in the human airway. Chest 2005;127:1999-2006.

-6 Cox PG, et al: Radiofrequency ablation of airway smooth muscle for sustained treatment of asthma: preliminary investigations. Eur Respir J 2004;24:659-663.

7 Martin L, Mayse ML, Rubin AS, Lampron N, Simof M, Duhamel D, Musani AI, Yung RC, Mehta AC: Clinical pearls for bronchial thermoplasty. J Bronchol 2007;14:115-123.

$>8$ Lee JA, Rowen DW, Rose DD: Bronchial thermoplasty: a novel treatment for severe asthma requiring monitored anesthesia care. AANA J 2011;79:480-483.

-9 Pawlowski J: Anesthetic considerations for interventional pulmonary procedures. Curr Opin Anaesthesiol 2013;26:6-12.

$>10$ Lo YL, et al: Feasibility of bispectral indexguided propofol infusion for flexible bronchoscopy sedation: a randomized controlled trial. PLoS One 2011;6:e27769.
11 Clark G, et al: Titrated sedation with propofol or midazolam for flexible bronchoscopy: a randomised trial. Eur Respir J 2009;34:12771283.

12 Chrissian AA, Bedi H: Bronchoscopist-directed continuous propofol infusion for targeting moderate sedation during endobronchial ultrasound bronchoscopy: a practical and effective protocol. J Bronchology Interv Pulmonol 2015;22:226-236.

13 Tschopp JM, et al: Titrated sedation with propofol for medical thoracoscopy: a feasibility and safety study. Respiration 2011;82:451457.

14 Carmi U, et al: Propofol safety in bronchoscopy: prospective randomized trial using transcutaneous carbon dioxide tension monitoring. Respiration 2011;82:515-521.

15 Bel EH, et al: Diagnosis and definition of severe refractory asthma: an international consensus statement from the Innovative Medicine Initiative (IMI). Thorax 2011;66:910917.

16 Bousquet J, et al: Uniform definition of asthma severity, control, and exacerbations: document presented for the World Health Organization Consultation on Severe Asthma. J Allergy Clin Immunol 2010;126:926938.

17 Chernik DA, et al: Validity and reliability of the Observer's Assessment of Alertness/Sedation Scale: study with intravenous midazolam. J Clin Psychopharmacol 1990;10:244251.

18 Langmack EL, et al: Serum lidocaine concentrations in asthmatics undergoing research bronchoscopy. Chest 2000;117:1055-1060.

19 Revill SI, et al: The reliability of a linear analogue for evaluating pain. Anaesthesia 1976; 31:1191-1198.
20 Mavritsin LE, Lifshits NA: Complications in the fiber bronchoscopy of bronchial asthma patients (in Russian). Klin Med (Mosk) 1980; 58:37-40.

21 British Thoracic Society Bronchoscopy Guidelines Committee, a Subcommittee of the Standards of Care Committee of the British Thoracic Society: British Thoracic Society guidelines on diagnostic flexible bronchoscopy. Thorax 2001;56(suppl 1):i1-i21.

22 Wechsler ME, et al: Bronchial thermoplasty: Long-term safety and effectiveness in patients with severe persistent asthma. J Allergy Clin Immunol 2013;132:1295-1302.

23 Bonta PI, et al: Conscious sedation for EUS of the esophagus and stomach: a double-blind, randomized, controlled trial comparing midazolam with placebo. Gastrointest Endosc 2003;57:842-847.

24 Goudra BG, et al: Anesthesia for advanced bronchoscopic procedures: state-of-the-art review. Lung 2015;193:453-465.

25 Hwang J, et al: Comparison of alfetanil and ketamine in combination with propofol for patient-controlled sedation during fiberoptic bronchoscopy. Acta Anaesthesiol Scand 2005;49:1334-1338.

26 American Society of Anesthesiologists Task Force on Sedation and Analgesia by Non-Anesthesiologists: Practice guidelines for sedation and analgesia by non-anesthesiologists. Anesthesiology 2002;96:1004-1017.

27 DeWitt JM: Bispectral index monitoring for nurse-administered propofol sedation during upper endoscopic ultrasound: a prospective, randomized controlled trial. Dig Dis Sci 2008; 53:2739-2745.

28 Bosslet GT, et al: Nurse-administered propofol sedation: feasibility and safety in bronchoscopy. Respiration 2010;79:315-321. 